\title{
A Learning and Helping Virtual Reality App Using Voice Frequency
}

\author{
Prabhat Ranjan Rai \\ Student, Department of \\ Computer Science Engineering \\ Galgotias University \\ Greater Noida, Uttar Pradesh, India \\ prabhatrai123450@gmail.com
}

\author{
Yash Gupta \\ Student, Department of \\ Computer Science Engineering \\ Galgotias University \\ Greater Noida, Uttar Pradesh, India \\ yashguptarules@gmail.com
}

\author{
Shubhangi Pandey \\ Student, Department of \\ Computer Science Engineering \\ Galgotias University \\ Greater Noida, Uttar Pradesh, India \\ pandeyshubhangi2001@gmail.com
}

\begin{abstract}
Virtual Reality is implemented by a combination of technologies that are used in order to visualize and provide interaction with a virtual environment.

Computer generated Reality is carried out by a blend of advances that are utilized to picture and furnish connection with a virtual climate. These conditions frequently portray threedimensional space which might be reasonable or nonexistent, plainly visible or infinitesimal and dependent on sensible actual laws of elements, or on fanciful elements. The large number of situations that VR might be utilized to portray make it comprehensively appropriate to the numerous regions in instruction. A vital element of VR is that it permits multi-tactile collaboration with the space being pictured. Here we take a gander at how this mix of multi-tactile perception and intuitiveness make VR unmistakably appropriate for compelling learning and attempt to clarify this viability regarding the benefits managed by dynamic learning through encounters.
\end{abstract}

This venture would make class intriguing just as innovative. Since most understudies can without much of a stretch explore augmented reality they feel achieved [1].

Analysts have investigated the advantages and uses of augmented reality (VR) in various situations. VR has a lot of potential and its application in schooling has seen a lot of examination interest recently [1]. Nonetheless, minimal orderly work presently exists on how analysts have applied vivid VR for advanced education purposes that considers the utilization of both very good quality and spending head-mounted presentations (HMDs) [1]. Thus, we propose utilizing orderly planning to distinguish plan components of existing exploration devoted to the use of VR in advanced education [2]. The looked into articles were gained by removing key data from reports recorded in four logical computerized libraries, which were sifted deliberately utilizing rejection, incorporation, self-loader, and manual techniques. Our survey underlines three central issues: the current area structure regarding the learning substance, the VR plan components, and the learning speculations, as an establishment for effective VR-based learning [3]. The planning was directed between application spaces and learning substance and between plan components and learning substance. Our examination has uncovered a few holes in the utilization of VR in the advanced education circle-for example, learning speculations were not frequently viewed as in VR application improvement to help and guide toward learning results [4]. Moreover, the assessment of instructive VR applications has fundamentally centered around convenience of the VR applications as opposed to learning results and vivid VR has generally been a piece of trial and improvement work instead of being applied consistently in real educating. By and by, VR is by all accounts a promising circle as this investigation recognizes 18 application spaces, showing a superior gathering of this innovation in numerous orders. The distinguished holes highlight neglected locales of VR plan for training, which could rouse future work in the field [5].

Despite the fact that VR isn't new, the new improvements in vivid advances - regarding representation and collaborations have made VR progressively alluring to researchers. The most recent VR head-mounted presentations (HMDs), like HTC Vive or Oculus Rift, permit clients to encounter a serious level of inundation.

Inundation portrays the inclusion of a client in a virtual climate during which their familiarity with time and this present reality frequently gets detached, along these lines giving a feeling of "being" in the errand climate all things being equal [6]. characterize this term as "a view of being genuinely present in a non-actual world by encompassing the client of the VR framework made with pictures, sound, or different boosts" so a member feels the individual is really "there" [6].

Keywords: Virtual Reality, Unity, Adobe Photoshop, Unreal Engine, Information Management, Education of informatics, Immersive Learning.

\section{INTRODUCTION}

In like manner, low-spending plan HMDs for cell phones, like Samsung Gear VR and Google Cardboard, empower everybody to encounter vivid virtual conditions. Moreover, the current gadgets likewise offer association abilities. While very good quality VR hardware accompanies devoted regulators that help game play (e.g., Oculus Touch), low-financial plan HMDs, like Google Cardboard, support look control or empower the client to interface with the virtual climate by giving an attractive switch [7].

The HMD market is relied upon to be esteemed at USD 25 billion by 2022, developing at a Compound Annual Growth Rate of $39.52 \%$ somewhere in the range of 2019 and 2025 (Global Augmented Reality, 2019) [7]. Hence, now is the ideal opportunity to contemplate vivid VR, essentially because of the expanded capacity of VR innovation and decreased expenses. For instance, the as of 
late delivered Oculus Quest comes as a cordless HMD. While the plan empowers the client to move all the more uninhibitedly, the expense is set at around 400 USDpractically a similar cost as the past age Rift with links [7].

Besides, VR has been portrayed as the learning help of the 21st century (Rogers, 2019). An examination proposes that understudies hold more data and can more readily apply what they had realized in the wake of partaking in VR works out (Krokos, Plaisant, and Varshney, 2019). Considering the potential learning upgrade through VR use, it is justifiable why specialists, associations, and instructors these days investigate this innovation seriously, hoping to add an additional measurement to the homeroom regarding both educating and learning [8].

VR can be defined as "the sum of the hardware and software systems that seek to perfect an all- inclusive, sensory illusion of being present in another environment" (Biocca\& Delaney, 1995). Immersion, presence, and interactivity are regarded as the core characteristics of VR technologies (Ryan, 2015, Walsh and Pawlowski, 2002). The term interactivity can be described as the degree to which a user can modify the VR environment in real-time (Steuer, 1995). Presence is considered as "the subjective experience of being in one place or environment, even when one is physically situated in another" (Witmer \& Singer, 1998) [8]. While researchers largely agree on the definitions of interactivity and presence, differing views exist on the concept of immersion. One branch of researchers suggests that immersion should be viewed as a technological attribute that can be assessed objectively (Slater \& Wilbur, 1997), whereas others describe immersion as a subjective, individual belief, i.e., a psychological phenomenon (Witmer \& Singer, 1998) [8].

\section{EASE OF USE}

- 3D Imagination: Talking about the immense extents of human creative mind, Virtual reality and Augmented reality can cook a tremendous extension in the application to designing in such after manners:

- Enabling specialists to see their undertaking in 3D and gain a more noteworthy comprehension of how it functions.

- Using perceptions and 3-D displaying instruments for making the ideal plan.

- Not just permitting the plan group to roll out important improvements in their task however saving both their time and cash as well.

- Predicted visuals of measurements and investigation for designing tasks.

- Used in Automobile and Aerospace tasks.

- So, a shift from computerized plan to augmented reality is set to change to the manner in which configuration engineers work. As there are different streams in designing. VR can be applied in every one of them bringing about beneficial yields.

- Virtual Learning:

i. Remote admittance to a limitless exhibit of instructive administrations (subjects and mentors) offered around the world

ii. Individualized learning measure that thinks about the individual degree of ability, singular requirements, and diverse learning styles

iii. Safe and secure learning climate

iv. Flexible learning regarding time, area, and speed

v. Cost-adequacy, time-viable, effectively versatile... and considerably more

- Virtual learning consolidates the entirety of the previously mentioned terms. It can defeat numerous downsides of the actual climate like time, offices, area, and so forth Online conditions permit instructors to work with bigger quantities of understudies and advance their standard undertakings. Virtual adapting additionally carries new academic strategies into the customary types of schooling and makes learning more customized and advantageous [8].

- Immersive Language Learning: Language inundation, or basically drenching, is a method utilized in bilingual language schooling in which two dialects are utilized for guidance in an assortment of themes, including math, science, or social examinations. The dialects utilized for guidance are alluded to as the L1 and the L2 for every understudy, with L1 being the understudy's local language and L2 being the subsequent language to be obtained through submersion projects and methods [9].

There are various sorts of language submersion that rely upon the age of the understudies, the classtime spent in L2, the subjects that are instructed, and the degree of support by the speakers of L1 [10]. In spite of the fact that projects contrast by country and setting, most language submersion programs have the general objective of advancing bilingualism between the two unique arrangements of language-speakers. By and large, biculturalism is likewise an objective for speakers of the lion's share language (the language verbally expressed by most of the encompassing populace) and the minority language (the language that isn't the larger part language) [10].

- Examination has shown that such types of bilingual schooling give understudies by and large more prominent language appreciation and creation of the L2 in a local like way, particularly more noteworthy openness to different societies and the protection of dialects, especially legacy dialects.

- Creating Better Learning Opportunities: Virtual reality can be utilized to upgrade understudy learning and commitment. VR instruction can change the manner in which instructive substance is conveyed; it deals with the reason of making a virtual world - genuine or 
envisioned - and permits clients see it as well as communicate with it. Being inundated in the thing you're learning rouses you to completely get it. It'll require less psychological burden to deal with the data [11].

- Here are only a couple properties that makes computer generated reality in training so incredible.

- $\quad$ Better feeling of spot

- When understudies read about something, they frequently need to encounter it. With VR, they aren't restricted to word portrayals or book delineations; they can investigate the subject and perceive how things are assembled [11].

- Thanks to the sensation of essence VR gives, understudies can find out about a subject by living it. It's not difficult to fail to remember that VR encounters aren't genuine - a body really trusts it's in another spot. This inclination connects with the psyche in a manner that is astounding.

- Unlimited Possibilities: It's a verifiable truth that individuals learn best by doing; nonetheless, on the off chance that you investigate present day training, you'll perceive how little adapting really occurs by doing [12]. Understudies are centered around perusing guidelines instead of utilizing them practically speaking. VR in training gives an encounter anchor to the guidance. With VR instruction, students are enlivened to find for themselves. Understudies have a chance to learn by doing instead of inactively perusing.

\section{A. Feasibility Analysis}

This Project will be under the financial plan of everyone since it doesn't cost a lot .

\section{B. Specialized Feasibility :-}

Programming projects which we will be creating utilizes the idea of AI, Adobe Photoshop and Java. So According to that it will cost not in excess of a Rs. 1,000/ - .

\section{Equipment Feasibilty :-}

The equipment that might be utilized in this undertaking will be Virtual Reality System that might be utilized and it will cost in excess of a Rs 90,000/ - . We will be likewise utilizing other framework to get the voice recurrence in this venture and that will cost in excess of a Rs 2000/-

\section{Spending Feasibility:-}

The all out financial plan of this task is a lot of costly and it can't be embraced by every single individual yet we'll make an honest effort to make it less expensive so everybody can manage the cost of that.

The Total expense of the task will be approx. Rs. 1,00,000/ - .

We will utilize every one of the quality items so it doesn't get harmed oftentimes and will be working for an extremely long haul. So we will be not trading off with the nature of the item.
There will be additionally the warrantee of this product and its parts for 1 year.

\section{E. Authors and Affiliations}

A few years ago, virtual reality (VR) in education was too far out in left field to even be considered feasible. It was too futuristic. The high cost of technology prevented using its capabilities to the full [13].

The early 60s saw the first device capable of creating a thorough virtual reality experience. Technology stalled. VR could only dramatically evolve with the appearance of specific VR helmets for a thriving gaming industry.

But now, virtual reality in education shows great promise. VR presents information in a way that aids perception through a three- dimensional model. When students need to digest huge portions of information [13].

No other technology today comes close to VR in significantly improving and simplifying the education process.

\section{F. Related Work}

A couple of months prior, we delivered our first augmented simulation application for Oculus Go and Samsung VR. Almost a large portion of a year really taking shape, we banded together with Oculus to build up a stage where we could convey the entirety of our substance in a more vivid manner. With the greater part of our substance being conveyed fundamentally on the web [14], it was significant for us to have a local application to house the entirety of our vivid substance. Notwithstanding, we likewise saw the application as a chance to encourage a greater quality driven and connecting with experience for our crowd. Maybe than zeroing in on particular VR pieces with one-off points, we made mixed media libraries dependent on topics and issues, including other related straight substance from Al Jazeera to offer our watchers a chance to see each issue in more profundity.

Building an augmented simulation application implied reexamining essential interface plans that we have all gotten acquainted with on portable applications. It implied failing to remember what we think about building 2-D applications for telephones, and applying fundamental standards of plan to a totally new stage.

It was a remarkable excursion, so we needed to share our learnings and the means we took to make our VR application, Al Jazeera Contrast [15].

\section{Stage 1:}

Characterize the objectives for the application

This may appear glaringly evident, however it's the main advance to consider prior to choosing to build up a VR application. You need to characterize clear objectives of what you need to accomplish with this application, regardless of whether it be an independent encounter, a multi-story stage, or a game. Without clear boundaries, you'll always be unable to make a perfect involvement in your application. 


\section{Stage 2:}

Choose what stages to plan for

Then, you need to choose which stage you need to create and plan the VR application for. Some significant inquiries to pose include: What gadget would you say you will fabricate your application for? Will be it for versatile, or will it be for a VR headset? On the off chance that versatile, will it be Android, iOS (or both)? Will your application remain solitary or live inside Oculus or other VR suppliers?

Stage 3:

Pick the engineers

For the improvement part of the application, there are two different ways you can go - you can fabricate the application in-house on the off chance that you have the important ranges of abilities and assets, or you can re-appropriate it to a specific advancement group.

\section{Stage 4:}

Design the look and style of the interface Designing for a VR application is not the same as planning for an ordinary portable application. A versatile application is intended to be agreeable in your grasp and is restricted by the bounds of the cell phone's screen. You are planning for a 2-D climate, and the cooperations are completely founded on the screen.

\section{Stage 5:}

Release the application and persistently improve it Your application is done and prepared for discharge. In spite of your innumerable preliminary attempts and careful testing, consistently be prepared and mindful should anything come up. Think about your application as something that isn't stale; it shouldn't simply be one-and-done. Maybe, begin arranging client input and joining their notes for future updates of the application; similarly as you gain from the client analyzers previously.

\section{G. Tools Used}

Unity

Amazon Sumerian

Google VR for everyone

Unreal Engine 4 (UE4)

CRYENGINE

Blender

3ds Max

SketchUp Studio

Maya

\section{H. Figures}

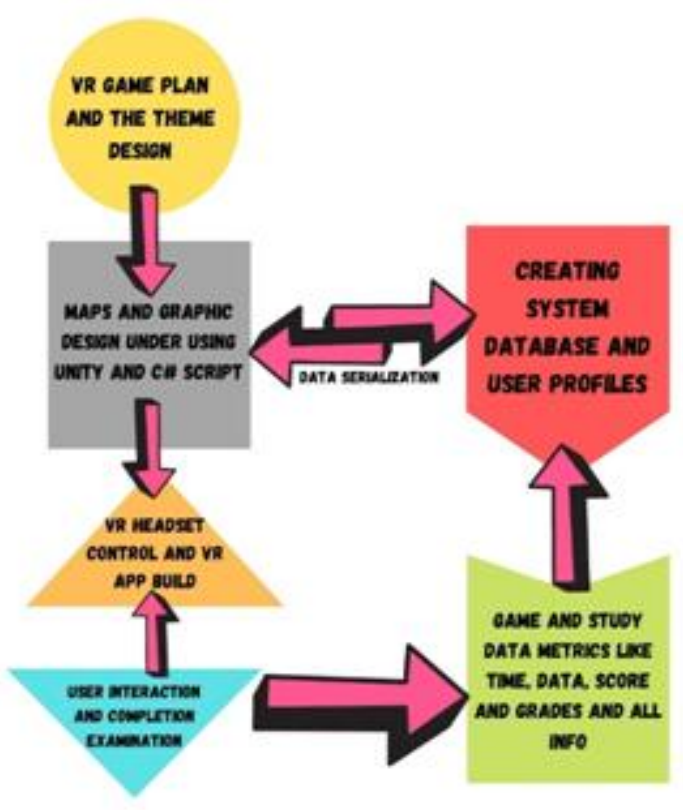

Fig. 1. Working Model

\section{CONCLUSION}

This project would make class interesting as well as creative. Because most students can easily navigate virtual reality they feel accomplished.

It will also help students to understand and feel the real life problem and they will get connected to the real world through the learning app.

It is beneficial for all ages people from student to old age as they get to visualize things then understand and learn.

\section{ACKNOWLEDGMENT}

I would like to express my special thanks of gratitude to my guide Mr. Anurag Singh as well as our university who gave me the golden opportunity to do this wonderful project on the topic "A learning and helping virtual reality app using voice frequency", which also helped me in doing a lot of Research and I came to know about so many new things I am really thankful to them.

\section{REFERENCES}

[1] End-Users' Augmented Reality Utilization for Architectural Design Review Year: 2020

[2] Virtual Reality and Its Applications in Education: Survey Year: 2019

[3] Designing an Interactive Learning Environment to Support Children's Understanding in Complex Domains Year: 1999

[4] The Future Of Edtech And Learning In India From An AR/VR Lens Year: 2020

[5] Virtual Reality: another world within sight Year: 2020

[6] 'An Eye-Opener': Virtual Reality Shows Residents What Climate Change Could Do Year: 2019

[7] Augmented Reality and Virtual Reality Market Year: 2020

[8] A systematic review of immersive virtual reality applications for higher education: Design elements, lessons learned, and research agenda Year: 2020 
[9] Promethean ClassFlow Announces New Digital Content Partnerships to Promote Collaborative \& Immersive Learning •Year: 2017 -Publication: Press Release, published in EducationDive.com • Full text: tba

[10] Developmentally distinct experiences among novice teachers reflecting on Teaching English as a Foreign Language (TEFL) Year: 2019

[11] Cyberspace: A new branch of international customary law? Author: Polański, Paul Przemysław •Year: 2017 •Journal: Computer Law \& Security Review: The International Journal of Technology Law and Practice, Volume: 33, Issue: 3, Pages: 371-381 •ISSN: 0267-3649 DOI: 10.1016/j.clsr.2017.03.007

[12] Universal Design for Learning Author: Basham, James D., Smith, Sean J., Hall, Tracey E. and Satter, Allyson L. •Year: 2016

[13] -Journal: Journal of Special Education Technology, Volume: 31, Issue: 3, Pages: 147-155 Full text: Free, PDF download available from journal web site

[14] Virtual Reality in Education: Your Ultimate List of 2021 Cannot-Miss Apps Year: 2020
[15] Oculus promises it hasn't forgotten the Gear VR Year: 2018

[16] Is immersive content the future of journalism? Year: 2018

[17] From e-learning to VR-learning: An example of learning in an immersive virtual world Author: Freina, L., Bottino, R. and Tavella, M. -Year: 2016

[18] -Journal: Journal of E-Learning and Knowledge Society, Volume: 12, Issue: 2, Pages: 101-113 •Full text: Free, PDF download available from journal web site $\cdot$ ISSN: 18266223

[19] The Internet as a New Tool in the Rehabilitation Process of PatientsEducation in Focus

[20] Author: Forczek, Erzsébet, Makra, Péter, Lanyi, Cecilia and Bari, Ferenc •Year: 2015

[21] -Journal: International Journal of Environmental Research and Public Health, Volume: 12, Issue: 3, Pages: 2373-2391

[22] -Full text: Free, Online and PDF download available from PubMed -ISSN: 1661-7827 